\title{
Mycological study of dermatophytosis in a part of South India
}

\author{
Raghavendra Rao Morubagal ${ }^{\star}$, Rashmi Padmanabha Mahale ${ }^{2}$, Sowmya Govindanahalli Shivappa ${ }^{3}$, Tejashree \\ Anantharajaurs ${ }^{4}$ and Madhuri Kulkarni ${ }^{5}$
}

\begin{abstract}
Department of Microbiology, JSS Medical College, Shivarathreshwara Nagar Mysore 570015, Karnataka, India. Email: getdrraghurao@yahoo.com
\end{abstract}

Received 20 June 2016; Received in revised form 18 July 2016; Accepted 25 July 2016

\begin{abstract}
Aims: Epidermophyton, Microsporum and Trichophyton are the genera of dermatophytes causing superficial mycoses. These infections are on rise due to increase in immunocompromised patients and favorable environmental conditions in countries like India. The present study was undertaken to identify dermatophytes causing superficial fungal infection by microscopy and culture techniques which helps in accurate diagnosis and appropriate treatment of cases.

Methodology and results: Samples were collected from affected sites after cleaning the affected surface with $70 \%$ alcohol. All samples were microscopically examined for presence of hyphal structures by digesting in $10 \%$ to $40 \% \mathrm{KOH}$ solution. All samples were inoculated into Sabouraud dextrose agar with chloramphenicol and Sabouraud dextrose agar with cycloheximide and chloramphenicol and incubated at room temperature for four weeks. Tease mount technique and slide culture technique were used for identification of dermatophytes. One hundred and ten samples from clinically suspected dermatophytoses which includes $77(70 \%)$ from male and $33(30 \%)$ from female patients were processed for identification of dermatophytes. Samples were subjected to microscopy and culture. In 61 samples (54.54\%) fungal hyphae were seen by direct microscopic examination (KOH). Fifty six samples (50\%) yielded dermatophyte growth in culture. Trichophyton rubrum was the predominant species isolated followed by $T$. violaceum and T. mentagrophytes.

Conclusion, significance and impact of study: Accurate and rapid diagnosis of superficial fungal infection is essential for proper management of cases. Direct microscopy is very good method for routine diagnosis, however culture remains gold standard.
\end{abstract}

Keywords: Dermatophytes, direct microscopy, culture, Sabouraud dextrose agar

\section{INTRODUCTION}

Dermatophyte infections are earliest known fungal infections affecting mankind and are very common throughout the world (Richa et al., 2012). Dermatophytes are aerobic fungi that infect the stratum corneum of the skin, the hair shaft, and the nail by producing enzyme proteases that digest keratin and allows colonization and invasion (Surendran et al., 2014).Traditionally infections caused by dermatophytes (ringworm) have been named according to the anatomical locations involved, by appending the Latin term designating the body site after the word Tinea, for example, infection of scalp is called tinea capitis (Hayand and Moore, 2004). Epidermophyton, Microsporum and Trichophyton are the genera of dermatophytes causing superficial mycoses. Dermatophytes are also classified according to their habitat, being either anthropophilic associated with humans, zoophilic associated with animals or geophilic associated with soil. Anthropophilic species are responsible for the majority of human infections and tend to be chronic with little inflammation. Infection caused by zoophiles and geophiles are associated with acute inflammation. Dermatophyte colonization is characteristically limited to the dead keratinized tissue of the stratum corneum and results in either a mild or intense inflammatory reaction. Although the cornified layers of the skin lack a specific immune system to recognize this infection and rid itself of it, nevertheless, both humoral and cell-mediated reactions and specific and nonspecific host defense mechanisms respond and eventually eliminate the fungus, preventing invasion into the deeper viable tissue. These organisms are assuming greater significance due to the excessive use of immunosuppressive drugs for controlling serious infectious as well as non-infectious conditions (Bhatia and Sharma, 2014).

The tinea infections are prevalent globally but they are common in tropics and may reach epidemic proportions in geographical areas with higher humidity, over-population and poor hygienic living conditions. In India due to hot and humid condition, dermatophytosis is very common superficial fungal infection (Niranjan et al., 2012). The distribution, frequency and the causative agents involved 
vary from place to place depending upon the climatic, socioeconomic conditions and the population density (Das et al., 2009). Measures for preventing dermatophytosis include practicing good personal hygiene, keeping the skin dry and cool at all times and avoiding sharing towels, clothing, or hair accessories with infected individuals. The treatment of dermatophytoses would be most appropriate when the selection of antimicrobial agent is based on the identity of the causative agent. For example, griseofulvin is effective only for dermatophytic infections, with no activity against Candida spp. And non-dermophytic molds. Terbinafine shows fungicidal activity against dermatophytes with a cure rate of 80 to $95 \%$ but shows only fungistatic activity against Candida albicans. For nondermatophytic molds infections, the role of terbinafine is not well defined and topical amorolfine lacquer may be effective for select patients (Denning et al., 1995). The present study was undertaken to identify dermatophytes causing superficial fungal infection by direct microscopy and culture techniques, which helps in appropriate treatment of cases and also throws light different species of dermatophytes prevalent in this part of India.

\section{MATERIALS AND METHODS}

Clinically suspected 110 cases of dermatophytoses attending the Out Patient Department of a tertiary care hospital were included in present study.

\section{Collection of samples}

Samples were collected after cleaning the affected surface with $70 \%$ ethanol in order to remove the dirt and environmental contaminants. From skin lesions, scales were collected from margins of the lesion with a sterile blunt scalpel, hair samples were plucked with sterile surgical forceps and infected nails using nail clippers. Samples were collected in paper envelope, labelled and transported to the microbiology laboratory.

For direct microscopy the sample collected was screened for the presence of filamentous, septate, branched hyphae by using potassium hydroxide mount $(\mathrm{KOH})$, which acts as keratinolytic agent and makes fungal hyphae visible. A $10 \% \mathrm{KOH}$ was used for hair samples, $20 \% \mathrm{KOH}$ for skin scrapings and $40 \% \mathrm{KOH}$ for nail samples. The sample (hair, skin and nail clipping) was placed on a clean glass slide and a drop of $\mathrm{KOH}(10 \%$ $40 \%$ ) solution was added and slide passed through a burner flame to hasten keratolysis. When keratolysis softened the sample, a clean glass cover slip was kept on the sample and pressed to prevent the formation of air bubbles. The sample was kept in $\mathrm{KOH}$ for a variable duration ranging from $5 \mathrm{~min}$ to $2 \mathrm{~h}$, depending upon the thickness of the sample.

For culture one set of Sabouraud dextrose agar with chloramphenicol and another with Sabouraud dextrose agar with cycloheximide and chloramphenicol were inoculated with clinical material. The inoculated agar slopes were incubated in room temperature and observed daily for growth. If no growth was noticed by four weeks of incubation, culture was considered negative and discarded.

Dermatophytes grown from clinical samples were identified by observing rate of growth, colony characteristics and microscopic morphologies by doing tease mount technique using lactophenol cotton blue stain and slide culture technique.

\section{RESULTS}

In the present study out of 110 clinically suspected dermatophytoses, $77(70 \%)$ samples were from male patients and $33(30 \%)$ were from female patients. Maximum numbers of samples $(27.27 \%)$ were from patients in the age group of 21-30 years. Age and sex wise distribution is shown in Table 1 and Figure 1.

$78(70.9 \%)$ skin, $17(15.45 \%)$ hair, $15(13.63 \%)$ nail samples were processed by direct microscopy using $\mathrm{KOH}$ mount and culture by inoculating SDA media. Results of $\mathrm{KOH}$ mount and culture are shown in Table 2 . Approximately half the number of samples yielded the growth of dermatophytes, whereas the remaining samples did not yield growth of dermatophytes but some samples yielded the growth of non-dermatophytes like Aspergillus, Fusarium and yeast like Candida especially from nail samples.

Trichophyton rubrum (31) was predominant dermatophyte isolated followed by $T$. violaceum and $T$. mentagrophytes. Different types of dermatophytes isolated from various clinical types are shown in Table 3.

Table 1: Age and sex wise distribution of cases.

\begin{tabular}{lccc}
\hline Age in years & Male & Female & Total \\
\hline $0-10$ & 5 & 2 & 7 \\
$11-20$ & 13 & 5 & 18 \\
$21-30$ & 24 & 6 & 30 \\
$31-40$ & 15 & 6 & 21 \\
$41-50$ & 11 & 8 & 19 \\
$>50$ & 09 & 6 & 15 \\
Total & 77 & 33 & 110 \\
\hline
\end{tabular}

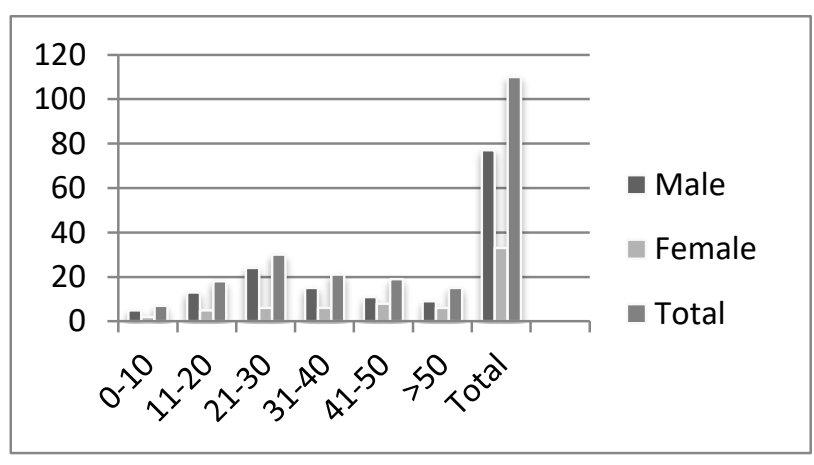

Figure 1: Bar chart showing age and sex wise distribution of cases. 
Table 2: $\mathrm{KOH}$ and culture results of the samples.

\begin{tabular}{llll}
\hline $\begin{array}{l}\text { Test } \\
\text { method }\end{array}$ & $\mathrm{KOH}$ positive & $\begin{array}{l}\mathrm{KOH} \\
\text { negative }\end{array}$ & Total \\
\hline $\begin{array}{l}\text { Culture } \\
\text { positive }\end{array}$ & 47 & 09 & $56(50 \%)$ \\
$\begin{array}{l}\text { Culture } \\
\text { negative }\end{array}$ & 14 & 40 & $54(49.09 \%)$ \\
\begin{tabular}{l} 
Total \\
\hline
\end{tabular} & $61(54.54 \%)$ & $49(45.45 \%)$ & $110(100 \%)$ \\
\hline
\end{tabular}

Chi square, $X^{2}=35.13 ; P<0.001$.

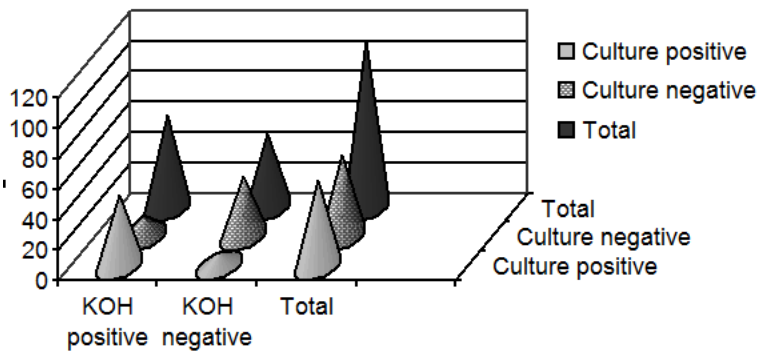

Figure 2: $\mathrm{KOH}$ and culture results of samples.

\section{DISCUSSION}

Earth has been documented as a natural territory for fungi which cover individual kingdom with evolution (Sharma et al., 2015). One fifth of the world's population suffers from mycoses (Bhadauria et al., 2001). The Mycoses caused by fungal infections of the skin, hair and nails are widespread and the most numerous group amongst all Mycoses. Primary infection starts through small skin break. These breaks are coming out through secretion of enzymes that digest keratin. The excreted enzyme plays a vital role in the process of infection and considered as primary virulent factors (Sharma et al., 2012). Apart from the clinical symptoms superficial fungal infections can cause debilitating effects on a person's quality of life. In India studies on dematophytosis has received increased attention of physicians and microbiologists in recent years due to the excessive use of immunosuppressive drugs for controlling serious infectious as well as non-infectious conditions and presence of favorable environmental conditions.

High incidence of dermatophytosis in present study was seen in the age group of 21-30 years. In studies

Table 3: Different types of dermatophytes isolated from samples.

\begin{tabular}{lllllll}
\hline Dermatophyte & $\begin{array}{l}\text { Tinea } \\
\text { corporis }\end{array}$ & $\begin{array}{l}\text { Tinea } \\
\text { Cruris }\end{array}$ & $\begin{array}{l}\text { Tinea } \\
\text { capitis }\end{array}$ & $\begin{array}{l}\text { Tinea } \\
\text { pedis }\end{array}$ & $\begin{array}{l}\text { Tinea } \\
\text { unguium }\end{array}$ & Total \\
\hline T. rubrum & 20 & 06 & 03 & 01 & 01 & $31(55.35 \%)$ \\
T. violaceum & 04 & - & 06 & - & - & $10(17.85 \%)$ \\
T. mentagrophytes & 04 & 02 & - & 02 & 01 & $09(16.07 \%)$ \\
T. tansurans & - & - & 02 & - & - & $02(3.57 \%)$ \\
Microsporum gypsium & 02 & - & - & - & - & $02(3.57 \%)$ \\
Epidermophyton floccosum & - & 02 & - & - & - & $02(3.57 \%)$ \\
Total & 30 & 10 & 11 & 3 & 2 & \\
\hline
\end{tabular}

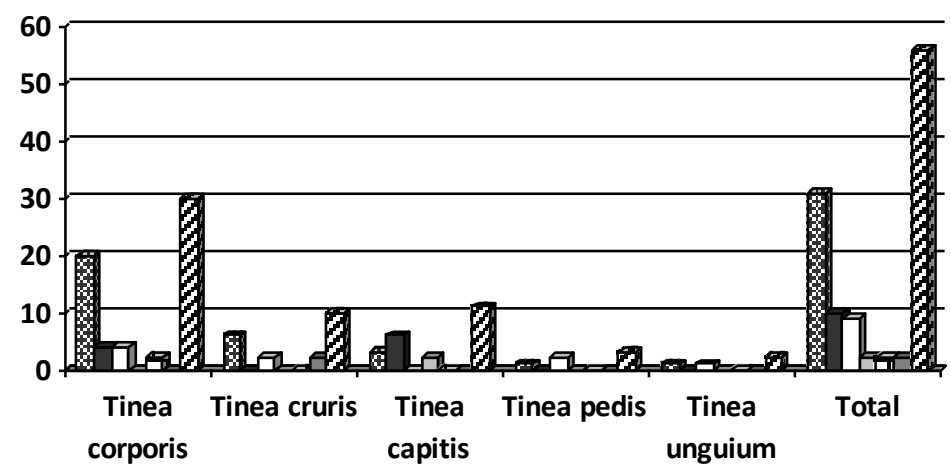

\begin{tabular}{|l|}
\hline T.rubrum \\
$\square$ T.violaceum \\
$\square$ T.mentagrophytes \\
$\square$ T.tansurans \\
$\square$ M.gypsium \\
$\square$ E.floccosum \\
$\square$ Total \\
\hline
\end{tabular}

Figure 3: Types of dermotophytes isolated.

done by Sen and Rasul (2006) and Sumana and Singaracharya (2004), the highest prevalence of dermatophytosis in the same age group were also reported. Increased incidence of dermatophytosis in younger age group is probably due to high physical activity which in turn increases the risk of exposure to 
fungal spores. About $70 \%$ of the samples in this study were from male patients. Similar observations were noted by other authors in their studies (Sumana and Singaracharya, 2004). Male predominance could be due to their increased outdoor activity when compared to females.

In the present study, 61 samples (54.54\%) were positive for fungal hyphae by direct microscopic examination $(\mathrm{KOH})$, whereas 56 samples $(50 \%)$ yielded growth of dermatophytes by culture. Forty samples (36.36\%) were both culture and $\mathrm{KOH}$ negative. The present study was supported by other studies. Singh and Beena (2003) reported microscopy positive in $60.38 \%$ of samples and culture in $44.6 \%$ of samples. About $53.38 \%$ samples did not show evidence of fungus either on direct microscopy or on culture. In a study done by Lakshmi et al. (2015) reported $\mathrm{KOH}$ positivity in $58.18 \%$ of samples and culture positivity in $56.36 \%$ of samples. In the present study culture positivity was more in $\mathrm{KOH}$ positive samples (83.92\%) compared to $\mathrm{KOH}$ negative samples (16.07\%). This difference is statistically significant $X^{2}=35.13$; $P<$ 0.001 . Similar findings were observed by Lakshmi et al. (2015). In their study, $85.9 \%$ samples were culture positive in $\mathrm{KOH}$ positive samples and $15.2 \%$ were culture positive in $\mathrm{KOH}$ negative samples. This shows that direct microscopy by $\mathrm{KOH}$ mount is a good screening test in the laboratory diagnosis of dermatophytosis.

Various tinea conditions in the present study were diagnosed by the clinician based on the clinical presentation. Tinea corporis was the most common clinical condition observed in which various exposed parts of the body are affected followed by tinea cruris and tinea capitis. Tinea conditions are as a result of exhaustive physical work and prolonged exposure to sun leading to excessive sweating. In addition, the tight fitting and synthetic clothing particularly in males provide damp, sweaty and warm skin conditions. All these factors favour the growth of dermatophytes. Tinea pedis and tinea unguium might result from wearing of socks and shoes for a long period providing damp conditions especially in inter-digital space. These findings were also reported by (Bhatia and Sharma, 2014).

Predominant species isolated in the present study was T. rubrum from $31(55.35 \%)$ samples .Other authors who reported $T$. rubrum as predominant isolate in their studies were Singh and Beena (2003) about $73.27 \%$, Surendran et al. (2014) about 67.5\% and Lakshmi et al. (2015) about $58.06 \%$. Second commonest species isolated in present study was $T$. violaceum, from $10(17.85 \%)$ samples. However, in contrast to present study other studies have reported $T$. mentagrophytes as second commonest organism (Bindu and Pavithran, 2002; Peerapur et al., 2004; Surendran et al., 2014). From tinea unguium cases only 2 Trichophyton species were isolated. However, five samples from tinea unguium cases yielded nondermatophytes, which includes two $C$. albicans, two Aspergillus niger and one Fusarium species which were not included in the Table 3 . It is a well-known fact that infections of nail is caused by dermatophytes as well as by yeasts and moulds like Aspergillus, Fusarium,
Curvularia etc. This could be one of the reasons for isolating less number of dermatophytes from nail samples in present study.

Neoscytalidium dimidiatum can cause clinical lesions resembling those induced by $T$. rubrum. Neoscytalidium spp. are moulds responsible for foot dermatomycoses in tropical and subtropical areas, and an increasing number of cases are being reported in temperate countries among immigrants from tropical areas. As there is no effective oral or topical treatment for skin and nail infections due to Neoscytalidium spp., accurate etiologic diagnosis is required to discriminate these infections from those due to dermatophyte species. Thus, improper antifungal treatments, often expensive and sometimes associated with drug toxicity, can be avoided (Kinda et al., 2012).

Identification of individual dermatophyte species causing infection is also important for several reasons. For example, $M$. canis commonly indicates a cat (rarely a dog) as a persistent inoculum source, while M. gypseum, causing similar lesions, indicates contact with contaminated soil. Second, the actual treatment regimens may differ for different dermatophyte species: for example, Trichophyton tonsurans in tinea capitis tends to require shorter treatment times than $M$. canis. The latter fungus to some extent evades drug exposure by forming arthroconidia outside the hair shaft, while the former forms arthroconidia inside the hair shaft where contact with conventional anti-fungal drugs is relatively high. Third, especially in onychomycosis, culture and species identification may be needed to distinguish dermatophytes from non-dermatophytic species causing dermatophytosis-like infection that does not respond to anti-dermatophyte therapy (Graser et al., 2008).

\section{CONCLUSION}

Dermatophytosis is one of the commonly encountered fungal infections in developing countries like India. Accurate and rapid diagnosis is essential for proper management of cases. Direct microscopy is very good method for diagnosis of superficial fungal infections in routine laboratory especially in resource poor setting, however culture remains gold standard which provide information about different species of dermatophytes prevalent in the region and also helps in discriminating infections resembling dermatophytosis like infections caused by Neoscytalidium spp.

\section{REFERENCES}

Bhadauria, S., Jain, N., Sharma, M. and Kumar, P. (2001). Dermatophytosis in Jaipur: Study of incidence, clinical features and causal agents. Indian Journal of Microbiology 41, 207-210.

Bhatia, V. K. and Sharma, P. C. (2014). Epidemiological studies on dermatophytosis in human patients in Himachal Pradesh, India. SpringerPlus 3, 134.

Bindu, V. and Pavithran, K. (2002). Clinico-mycological study of dermatophytosis in Calicut. Indian Journal of 
Dermatology Venereology and Leprology 68, 259-261.

Das, K., Basak, S. and Ray, S. (2009). A study on superficial fungal infection from West Bengal: A brief report. Journal of Life Sciences 1(1), 51-55.

Denning, D. W, Evans, E. G. V., Kibbler, C. C., Richardson, M. D., Roberts M. M., Rogers, T. R., Warnock, D. W. and Warren, R. E.(1995). Fungal nail disease: A guide to good practice (report of a working group of the British Society for Medical Mycology). British Medical Jourmal 311, 1277-1281.

Graser, Y., Scott, J. and Summerbell, R. (2008). The new species concept in dermatophytes-a polyphasic approach. Mycopathologia 166(5-6), 239-256.

Hay, R. J. and Moore, M. (2004). Mycology. In: Rook's Textbook of Dermatology. Burns, T., Breathnach, S., Cox, N. and Griffiths, C. (eds.). 7th edn. Blackwell Science, London. pp. 31.1-31.101.

Kinda, A. B., Jean-Luc, B., Claire, L., Martine, F., Brunhilde, D., Gilles, Q., Noura, H., Xavier, N. and Marie-Elisabeth, B. (2012). Successful identification of clinical dermatophyte and Neoscytalidium species by matrix-assisted laser desorption ionization-time of flight mass spectrometry. Journal of Clinical Microbiology 50(7), 2277-2281.

Lakshmi, V., Jyothi, P. I. and Sai, L. K. (2015). Clinicomycological study of dermatophytosis in South India. Journal of Laboratory Physicians 7(2), 84-89.

Niranjan, H. P., Padmaja, N. and Priyanka, B. V. (2012). Study of onychomycosis at a tertiary care hospital in South India. Journal of Evolution of Medical and Dental Sciences 1(5), 823-829.

Peerapur, B. V., Inamdar, A. C., Puspha, P. V. and Shrikant, B. (2004). Clinicomycological study of dermatophytosis in Bijapur. Indian Journal of Medical Microbiology 273-274.

Richa, S, Nakuleshwar, D. J. and Suresh, S. (2012). Clinical and mycological study of dermatophytosis in Jaipur (India). International Journal of Pharmacy and Pharmaceutical Sciences 4(3), 215-217.

Sen, S. S. and Rasul, E. S. (2006). Dermatophytosis in Assam. Indian Journal of Medical Microbiology 24(1), 77-78.

Sharma, A., Chandra, S. and Sharma, M. (2012). Difference in keratinase activity of dermatophytes at different environmental conditions is an attribute of adaptation to parasitism. Mycoses 55(5), 410-415.

Sharma, V., Kumawat, T. K., Sharma, A., Seth, R. and Chandra, S. (2015). Distribution and prevalence of dermatophytes in semi-arid region of India. Advances in Microbiology 5, 93-106.

Singh, S. and Beena, P. M. (2003). Comparative study of different microscopic techniques and culture media for the isolation of dermatophytes. Indian Journal of Medical Microbiology. 21(1), 21-24.

Sumana, V. and Singaracharya, M. A. (2004). Dermatophytosis in Khammam. Indian Journal of Pathology and Microbiology 47(2), 287-289.

Surendran, K. A. K., Ramesh, M. B., Rekha, B., Nandakishore, B. and Sukum, D. (2014). A clinical and mycological study of dermatophytic infections. Indian Journal of Dermatology 59(3), 262-267. 